\title{
NOTAS
}

\section{Pasión y Verdad en el Teatro de Villaurrutia}

$\mathrm{D}$ ENTRO de la moderna literatura mexicana el teatro de Xavier Villaurrutia $^{1}$ tiene un doble interés. En primer lugar, simboliza el momento crítico de la evolución del teatro en México que su autor presenció. Es decir, la aparición de La biedra, en I94I, cierra en gran parte el ciclo de teatro experimental que había ido desarrollándose bajo influjos predominantemente extranjeros y se inicia la apertura del teatro mexicano hacia el gran público. Por esta razón La biedra le valió a Villaurrutia sendas cartas de J. L. Martínez y C. Gorostiza, ${ }^{12}$ aquél hostilizándole por su traición a los ideales del experimentalismo que hasta entonces había profesado; éste, más previsor, alegando un nuevo enfoque del teatro con miras al éxito de taquilla, afirmación que después hubo de afianzar en un reciente volumen de Teatro mexicano del siglo $\mathrm{XX}$.

Pero además de este interés general, el teatro de Villaurrutia tiene otro más particular. Se trata del curioso fenómeno de distanciamiento que aparentemente existe entre el dramaturgo y el poeta. Pues si bien, según Torres-Rioseco, la poesía de Villaurrutia está escrita "como si el corazón del poeta no vibrara con ninguna pasión", 3 en la mayor parte de su teatro predomina la nota pasional. Desde su primera pieza, Parece mentira (I934), se afirma la pasión como criterio de la existencia mis-

1 Para una discusión más general de la obra dramática de Villaurrutia y para sus datos biográficos remitimos al lector a V. F. Beck, "Xavier Villaurrutia, dramaturgo moderno", Revista Iberoamericana, XXXV (1952), pp. 27-39.

2 J. L. Martínez, Literatura mexicana del siglo XX (México, 1949), I, 135.

3 A. Torres-Rioseco, Ensayos sobre literatura bispano-americana (México, 1953), p. 204. 
ma. Aquí, en el repentino acceso de celos del marido, se efectúa el paso de serenidad a pasión. El mismo nos expone su situación - "Un ser dichoso, conforme e ignorante de cualquier tormento... hace veinticuatro horas habría podido decirles que ese hombre era yo. El bienestar, la comodidad, el desahogo económico y una alegría bien dosificada dentro y fuera de mí. Y no digamos un tormento, ni la más leve preocupación ensombrecía mis pensamientos ni mis costumbres..." Pero es el empleado quien, con la voz de Villaurrutia, subraya el sentido de esas afirmaciones, observando fríamente - "En una palabra: Vd. no existía". La frase es rotunda y no deja lugar a dudas: según el empleado, la única vida auténtica es la pasional. Sin la pasión no es uno más que espectador, un ser que vive de vida prestada, mientras el hombre presa de la pasión vive de veras - "empresario de un espectáculo en el que setá al mismo tiempo el actor y el espectador". La pasión le otorga señorío sobre la vida, la cual, gracias a su renovada y aumentada capacidad de acción, puede ahora "labrar a su antojo". Señalemos de paso que el advenimiento de la pasión no aniquila por completo el elemento de autoobservación. Más bien da lugar a un curioso desdoblamiento en el individuo - actor a la vez que observador de sí mismo- desdoblamiento que tipifica al personaje villaurrutiano.

Después de Parece mentira, esta potencia vitalizadora que Villaurrutia atribuye a las pasiones humanas: amor, odio o celos, prevalece en su teatro. Su influjo cómico, menos importante, se nota en el próximo 'auto profano' ¿En qué piensas? (I934) y más adelante en El pobre barba azul (1947). Las dos piezas muestran un claro lazo de parentesco entre sí, ya que ambas recalcan, si bien parodiándola, la teoría de la importancia vital de la pasión enunciada antes. En la primera hallamos en seguida un eco de lo expresado en Parece mentira:

Maria Luisa: "...Vd. no me ha amado nunca, $\mathrm{Vd}$. no me ana, luego..."

Ramón: "No existo".

Maria Luisa: "Eso es".

Otra vez, atenuada desde luego por el humorismo de la pieza, pero todavía evidente, estamos frente a la identificación de vida y pasión. Aún más estilizada, en El pobre barba azul, es de nuevo la pasión amorosa la que obra una sorprendente transfiguración vital.

Pero hemos de volver la mirada a las cinco piezas serias de Villa-

4 Xavier Villaurrutia, Poesía y teatro completo (México, 1953); p. 204.

5 Ibid., pp. 108-9. 
urrutia para ver el imperio de la pasión en toda su extensión. Empecemos con Teresa en La biedra. El título mismo de la obra, cuyo significado nos lo explica Ernesto, llama la atención sobre el papel que desempeña la pasión en la existencia de esta mujer. Sólo abrazándose a la pasión -primero por su marido, y luego por su hijastro-alcanza vida auténtica. Vive, nos dice Ernesto, de lo que abraza. Así, cuando se siente amenazada por el amor indudablemente más legítimo de Alicia, es con el grito característico de "Ahora me siento vivir más que nunca antes" 6 con el que reivindica su renovada pasión.

En La mujer legítima (1943) no es menor la intensidad emocional, si bien en estè caso no se puede hablar de pasión vital. Aquí, como en Juego peligroso (I950), la pasión aparece sencillamente como agente de la maldad connatural a nuestro modo de ser. En Marta asoma aquel "algo demoníaco" que señaló Raúl Leiva7 en el fondo del alma de su amigo Villaurrutia. No hay aquí ningún deseo de justificar la pasión por sus resultados, ni el sufrimiento por su aportación al espíritu. Tanto Sara como Teresa salen de la escena con su vida hecha trizas y sin la posibilidad de rehacerla. Van hacia la soledad. La lección de estas dos piezas es obvia: en ellas vemos claramente la visión pesimista de la naturaleza humana que forma como un sedimento en la personalidad del autor.

También estas dos piezas constituyen, ya de por sí, un ciclo en la producción dramática de Villaurrutia. En ellas la pasión, aun siendo lo más íntimamente vital que experimentamos, es una fuerza ciega, fatidica, que acarrea, como veremos, una comprensión angustiosa. Pero, en los últimos años de Villaurrutia, hay una tentativa de equilibrar la pasión y sus resultados, aunque la justificación de aquélla nunca llega a ser total. La intervención de Román en El yerro candente (I945) deshace el matrimonio de Eduardo e Isabel, revelando la debilidad de ésta, del mismo modo que, anteriormente, la decepción urdida por Marta había descubierto la ignobilidad de Rafael, pero halla su compensación reforzando el lazo que une a Antonia con su supuesto padre. Aquí por excepción Villaurrutia se sobrepone a su propia sensibilidad fundamental y se muestra "capaz de equilibrar con la substancia ideal la forma de la realidad contingente", capacidad, según Torres Bodet, 8 imprescin-

6 Ibid., p. 221.

7 R. Leiva, Imagen de la poesía mexicana contemporánea (México, 1959), p. 163.

8 J. Torres Bodet, "Homenaje a Benavente", Contemporáneos, IX (1929), p. 182. 
dible en un dramaturgo. También en Juego peligroso, su último drama, se salva la felicidad, pero a costa de lo que indica Arturo cuando dice al caer el telón: "Para volver a encontrarnos tú y yo ha sido necesario martirizarnos y martirizar a otros ... inocentes". ${ }^{9}$ No está lejos el mundo tenebroso y cruel de las producciones anteriores.

Con esto, pues, resulta evidente la gran diferencia que media entre el poeta y el dramaturgo en Villaurrutia. E. Abreu Gómez, aceptando el juicio antes citado de Torres-Rioseco, dice de la poesía de nuestro autor que "Sus figuras ... se mueven con precisión. En ellas la voz y el eco trenzan justo diálogo ... discurren con habilidad dentro del espacio que, previamente, $\mathrm{X}$ avier les ha creado. Allí quedan libres dentro de su prisión. Tienen libertad para todo menos para huír. $Y$ así ninguna salta impulsada por la savia encendida de la libertad. Para saltar les falta energía cósmica, conciencia heroica, validez de suicidio vital"..$^{10}$ Este juicio tiene que rectificarse con respecto a su teatro. En él, con excepción de Invitación a la muerte, de la que trataremos más adelante, es energía, la feroz energía de la pasión, lo que sobra, desborda, y produce más de un trágico desenlace.

Volviéndonos de nuevo a Parece mentiva, nuestro punto de partida, anotemos un singular aspecto del carácter del empleado, portavoz típico de las piezas de Villaurrutia en las que tantas veces el autor discurre por su propia cuenta. El empleado, mientras explica su teoría de la pasión al marido (y al auditorio), se muestra envidioso ante la angustia de éste y lamenta su propia posición de inferioridad. Ahora, basta recordar que este personaje es, él mismo, un creador poeta y novelista -uno, como dice, que vive miserablemente como un ladrón de los trozos de vida que roba a los demás, para darse cuenta de algo muy importante para la comprensión de Villaurrutia. A saber, que para él ser creador implica en algún modo encontrarse uno dotado de cierta capacidad de abstracción, de fría intuición, de inteligencia ${ }^{11}$ que dificulta toda manifestación de pasión en su propia vida. Parece ser, según cuentan cono. cidos suyos, que Villaurrutia era un hombre de frases lapidarias, de filosas ironías, un secuaz de Wilde que no desperdició su talento, en suma: todo lo opuesto de un pasional. He aquí el testimonio de su poesía: todo parece subrayar la dolorosa represión en él de verdadera capacidad de violenta emoción. Amante de la muerte, de la no-existencia,

- Xavier Villaurrutia, op. cit., p. 535.

10 E. Abreu Gómez, Sala de retratos (México, 1946), p. 292.

11 Véase el artículo de A. Magaña Esquivel, "Villaurrutia y. la inteligencia" en Sueño y realidad del teatro (México, 1949). 
Villaurrutia, que ya había identificado pasión y vida, rechaza aquélla a la vez que la mira con invencible nostalgia. Es curioso cotejar unas palabras del empleado a propósito de esa su vida sin pasión: "una realidad vacía, un mundo deshabitado, un camino sin paisaje, un sueño sin ensueños: en una palabra una muerte eterna", ${ }^{12}$ con la atmósfera de las poesías de Villaurrutia y, sobre todo, con el modo de ser de Alberto en Invitación a la muerte.

Esta obra, precisamente por ser la más personal que ha escrito, exploración previa de su propia personalidad antes de adentrarse en el resto de su teatro (ya que fué escrita en 1940), nos facilita no poco la comprensión de las comedias posteriores. Pues en todas ellas, al lado del elemento pasional, Villaurrutia introduce motivos, sobretodo el de la soledad, que se relacionan intimamente, a través de Invitación a la muerte, con los temas predilectos de su poesía.

Salvador Reyes Nevares, en unas páginas magistrales, quizás las mejores que se han escrito sobre Villaurrutia, ${ }^{13}$ procura definir el significado del amor para el poeta, analizando dos poesías suyas: "Nocturno de la alcoba" y "Amor condusse noi ad una morte". En palabras que igualmente pudieron haberse escrito teniendo presente sólo Invitación a la muerte, precisa Reyes Nevares que para Villaurrutia ell amor se funda en el afán de unirse con el amado mediante la posesión integral ontológica ("Un querer saber todo lo tuyo") que llega así a la substancia.y abandona lo vacilante y precario de la vida del ser singular. La raíz de la sensación de la muerte que acompaña siempre al amor en Villaurrutia es la percepción clara y continua de la imposibilidad de tal posesión total, tal unión e identificación perfectas. Es decir: la percepción de la radical soledad del individuo. Este complejo de preocupaciones (amor -muerte-soledad) producto, no de la pasión sino de la inteligencia obrando sobre la pasión, domina la concepción de Invitación a la muerte.

Se trata, como en otras obras de Villaurrutia, del descubrimiento de una verdad, en este caso por medio del estudio de una aguda crisis mental. No hay apenas acción; pero por eso mismo la obra ofrece mayor tiqueza de datos al crítico, ya que el diálogo, que así viene a ser el máximo sostén de la pieza, adquiere mayor intensidad conceptual. Se acrecienta la marcadísima tendencia de Villaurrutia a explicar sus dramas desde la escena, a subrayar lo que debiéramos comprender. Significativa

12 Xavier Villaurrutia, op. cit., p. 91.

13 S. Reyes Nevares, "La poesía amorosa de Xavier Villaurrutia" en $E l$ amor y la amistad en el mexicano (México, 1952). 
aquí es la presencia, al lado del principal observador y comentador de la acción (el joven Horacio), del médico, cuyo papel es análogo. La verdad que ellos nos señalan es, en este caso, de índole puramente intelectual. Alberto es un hombre que sufre por su propio modo de ser y que busca una salida de su angustia tratando de evadirse precisamente de sí mismo. La raíz de su personalidad no es otra que aquella intuición de la inevitable soledad humana, que se expresa maravillosamente en "Nocturno de la alcoba" y tantas otras poesías de Villaurrutia. Alberto, pues, como el empleado de Parele mentira, ilustra ese aspecto de la sensibilidad villaurrutiana que contrasta tan singularmente con la concepción de las obras siguientes: el sentimiento de incapacidad pasional y el correspondiente rechazo de la pasión y de toda emoción. Albetto rehuye la pasión, el amor, simbolizados por Aurelia, como también, al final renuncia a la emoción filial, pues los dos significan un atentado contra la soledad. El descubrimiento que hace, a través de su huida frustrada de sí mismo y de los demás, es que no hay solución para él sino la soledad: aquella muerte en vida que es el encontrarse totalmente a solas con su propia conciencia.

A primera vista Invitación a la muerte queda muy distanciada de las obras que siguen. No sólo falta la pasión, sino que ésta está conscientemente eliminada. En su lugar figura un problema psicológico que en las últimas escenas enlaza con la metafísica, con el enigma del destino del hombre. Otro tanto podríamos decir con respecto a la forma. No hay en los episodios aquel riguroso encadenamiento que ha hecho hablar de "precisión de relojería". 14 Alguno de ellos (v. gr. el de la dama loca) no es más que un pretexto para cambiar de tono durante algunos minutos sin apartarse del tema central. En el diálogo predomina un genuino humorismo, la risa sintomática de la angustia, muy lejos del persiflage de El pobre barba azul o las primeras escenas de Juego peligroso. Es la pieza más sincera, más auténtica, de Villaurrutia, obra por lo tanto esencialmente subjetiva y en ciettos aspectos inaccesible al público. En efecto, Invitación a la muerte representa la máxima incursión de la inteligencia de Villaurrutia, de su consciente actitud vital, en su teatro. Por eso queda algo aislado. Sin embargo su presencia abre una nueva perspectiva. Cabe sospechar que la pasión que desborda en las obras siguientes sea en realidad pasión reflejada, vicaria, nostalgia de pasión, por parte de aquel Villaurrutia, creador de Alberto y del empleado, cuya excesiva facultad analítica aplicada a sus propios sentimien-

14 J. L. Martinez, op. cit., p. 113 
tos se exterioriza en sus frías poesías, desnudas de vibración emocional. Mientras tanto subsiste soterrada la visión honda e intelectual de Invitación a la muerte.

Así es, efectivamente. Por poco que buceemos dentro de los dramas escritos después, encontramos, disimulado bajo las pasiones que se debaten en el primer plano, un fondo de frío pensamiento. Se asoma sobre todo en el tema, común a todos, de la búsqueda de la verdad. Valioso ejemplo de la importancia de este tema se nos of rece en el momento menos pensado, en las últimas escenas de El pobre barba azul, con aquel inesperado cambio de tono que concluye la acción. De repente se intercala un elemento patético y conmovedor, del todo extraño al desenfadado humorismo del resto de la pieza: el re-descubrimiento de "su verdad" por parte de Samuel. El diálogo de esta octava escena del tercer acto evoca conceptos ya familiares. En primer lugar, la verdad como sinceridad, es decir, como medio de aquel conocimiento integral de otra persona a que nos referimos arriba. Aun dentro de Invitación a la muerte, en donde la verdad subyacente es de orden más abstracto, asoma por momentos la preocupación por aquella otra verdad más humana. Señaladamente en la escena séptima del segundo acto: Alberto, en vez de definir su malestar en términos de soledad, habla aquí más bien de una duda que él no tiene valor de resolver. Pero luego vemos que no se trata de una duda auténtica, sino de una verdad demasiado amarga: "No es la verdad lo que busco, porque la verdad la tengo ya, o creo tenerla, al menos, aquí dentro como un vacío sin fin, en el pecho; no es la verdad sino la busca de la verdad y la mezquina comprobación de esa verdad fuera de mí en una palabra, en un gesto, en una situación". ${ }^{15}$ Concretamente se refiere a la conducta de su madre, uno de los muchos personajes del teatro villaurrutiano que representan no ya la maldad, sino lo innoble, y sobre todo, la insinceridad. Aquí, en $E l$ pobre barba azul, es la revelación de la insinceridad de Carmen ("tu verdad" como dice Samuel) lo que precipita el patético desenlace. Otro tanto ocurre en La biedra con respecto a Hipólito. Si bien, en efecto, es posible equiparar en Villaurrutia soledad y muerte, igualmente hay que identificar sinceridad y verdad.

El segundo concepto, en el que alcanzar la verdad equivale a "despertar", es también una constante de la obra de Villaurrutia y enlaza esta escena de $E l$ pobre barba azul, a primera vista tan marginal, con lo

15 Xavier Villaurrutia, op. cit., p. 315. 
más fundamental de su pensamiento. Para ver en qué medida esta penosa e involuntaria vuelta a la triste realidad por parte de Samuel refleja el proceso vital de su creador, basta recordar cuántas poesias de éste giran alrededor de la fórmula soñar-vivir / despertar-morir; tanto más cuanto que Samuel despierta a la soledad de una vida sin pasión, lo cual para Teresa significaba "volver a morir".

Así llegamos a la verdad como soledad: aquella soledad inevitable que aqueja a Alberto y que es, para Villaurrutia, parte integral del destino humano. Al final de la comedia todos se apartan simbólicamente, dejando solo a Samuel. Igualmente La biedra y La mujer legitima terminan en la soledad. Sobre todo en el personaje de Teresa, de la primera, el tema de la soledad contrapuesto al del amor muestra cómo confluyen en la obra dramática de Villaurrutia estas dos tendencias de su sensibilidad: goce de pasión por una parte, y por otra, mediata pero ineluctable visión del aislamiento individual. En el punto más dramático del tercer acto Teresa misma llega a puntualizar exactamente su anhelo: "me condenaron a la soledad y ... en esa soledad cada día más intensa, cada día más profunda, aprendí a amar ... no voy a abrir las manos y dejar escapar mi tesoro". ${ }^{16}$ Pero no puede eludir su destino y, como Samuel, pide que la dejen sola frente a "la soledad que ya veo abrirse delante de mí como una sombra y en la que entraré sola, para siempre". 17 Mientras tanto el último grito de su rival, Alicia, recalca su triunfo: "¡Si no estoy sola! ¡Si ya nunca estaré sola!" Sara también, al final de La mujer legítima, vuelve a la soledad de donde vino, reconociendo inútil su tentativa de integrarse a la familia de Rafael. Efectivamente, tanto en estas dos piezas como en las posteriores, la fórmula es la misma: 'por el sufrimiento hacia la verdad'. Cada uno de los dramas de Villaurrutia encierta una crisis que pone de manifiesto una nueva dimensión de la realidad, antes ignorada. Valgan como ejemplos la frase pronunciada por Teresa al final de La biedra: "Todo ha cobrado su verdadero sentido para mí ahora", y el suspiro del pobre Samuel: "Ahota despierto, y para siempre". Ambos acaban de reconocer "la verdad que, puesta delante de nosotros como un espejo implacable, nos da miedo $y$ nos hace gritar". ${ }^{18}$

Es también en La mujer legítima donde vemos más claramente el

16 Ibid., p. 220.

17 Ibid., p. 229.

18 Ibid., p. 224. 
último aspecto de la "verdad" villaurrutiana: verdad como maldad afbitraria, ya que todo el interés de la pieza está concentrado en Marta y en su lucha para destruir la felicidad de Sara y de su propio padre. Sería fácil acusar a Villaurrutia de incurrir aquí en la peor falta del dramaturgo que es, según Pérez de Ayala, ${ }^{19}$ el construir su enredo a base de personajes simpáticos y personajes antipáticos en vez de justificarlos a todos. Sara es simpática, noble, comprensiva; sus propias cualidades hacen más fácil la tarea de su adversaria, subtayando así el sometimiento del bien al mal que caracteriza esta fase de la producción de Villaurrutia. Marta es antipática, más aún que Rafael, su contrafigura masculina en Juego peligroso, más aún que Alicia (de La biedra), puesto que estos dos tienen en parte la justificación de su amor. Precisamente por la carencia de adecuada justificación en el caso de Marta, Villaurrutia echa mano al recurso, algo débil, de incipiente locura. Pero antes de aplicar con demasiado rigor el criterio de Ayala hagamos una pausa para considerar lo que implica la presencia de estos personajes tan esencialmente villaurrutianos. La presencia de auténticos malvados en el teatro de Villaurrutia no se debe, desde luego, a supervivencias teatrales de la época anterior. Toda su vida combatió el teatro de astracán. Nadie como él conocía al dedillo las tendencias modernas, entre ellas la desaparición del malvado como resorte dramático. Aquí, pues, se trata de sus convicciones intelectuales, de observador de la vida, exteriorizadas no sólo en la concepción de sus mejores dramas en términos de una abierta lucha entre el bien y el mal, sino también en toda aquella serie de portavoces que empieza con el empleado de Parece mentirc y de la cual Ernesto, en $L a$ biedra, es un ejemplo típico. En las afirmaciones de éste ("A la fealdad de las cosas y a la maldad de las personas no se resigna uno jamás"; "nunca hacemos el bien sin producir inevitablemente el mal en torno nuestro") reconocemos fácilmente el acento de su creador. Villaurrutia cree en la maldad, en la intrusión inevitable del mal en nuestra vida.

Entre estos límites se cierra el círculo de la sensibilidad dramática de Villaurrutia, construida ésta en partes iguales por aquella "luz fría de la inteligencia" que según J. L. Martínez caracteriza su poesía, y por una singular valorización de la pasión. Habría sido tentador escindir su personalidad literaria en dos secciones independientes entre sí, una poé-

19 Ramón Pérez de Ayala, Troteras y danzaderas (Buenos Aires, 1942), p. 119. 
tica y dramática la otra. Pero, tratándose del hombre, tal división es siempre arbitraria y artificiosa. Por ello la hemos rechazado. Sólo unificando las diversas facetas de su obra podemos lograr un juicio válido y equilibrado de la aportación dramática de Xavier Villaurrutia.

University of Glasgow

DONALD L. SHAW 